\title{
Genome-wide off-targets of drugs: risks and opportunities
}

\author{
Anil K Giri • Aleksandr Ianevski • Tero Aittokallio
}

Received: 1 August 2019 / Accepted: 11 August 2019/Published online: 20 August 2019

(C) Springer Nature B.V. 2019

Most approved drugs and small-molecule compounds modulate their therapeutic and toxic effects through multiple protein targets and biological pathways. Proteome-wide compound-target interaction networks (among both on- and off-targets) are therefore essential resources to better understand and make use of such polypharmacological effects of promiscuous compounds (Knight et al. 2010). Interestingly, systematic bioactivity profiling has revealed that some compounds may have higher potency against their unintended off-targets, compared to the primary on-targets (Tang et al. 2018). Unexplored off-target potencies of approved drugs may therefore lead to novel drug repurposing opportunities, i.e., finding new uses or targets for existing drugs (Pemovska et al. 2015; Kuenzi et al. 2019). A prime example of the utilization of compound-target interaction networks comes from finding synergistic compound combinations that lead to increased efficacy. For instance, the

A. K. Giri · A. Ianevski · T. Aittokallio

Institute for Molecular Medicine Finland (FIMM), University of Helsinki, FI-00290 Helsinki, Finland

A. Ianevski $\cdot$ T. Aittokallio

Helsinki Institute for Information Technology (HIIT), Aalto

University, FI-02150 Espoo, Finland

T. Aittokallio $(\bowtie)$

Department of Mathematics and Statistics, University of Turku, FI-20014 Turku, Finland

e-mail: tero.aittokallio@helsinki.fi recent AstraZeneca-Sanger Drug Combination DREAM Challenge benchmarked a number of machine learning algorithms in terms of their accuracy to predict synergistic drug pairs and associated biomarkers for 910 drug combinations across 85 cancer cell lines (http://dreamchallenges.org/initialresults-from-the-astrazeneca-sanger-drugcombination-prediction-challenge-presented/). Among 160 participants, all the winning methods incorporated prior knowledge of putative drugtarget interactions (Menden et al. 2019). However, proteome-wide target profiles are not important merely when exploring therapeutic potential, e.g., synergistic drug pairs, but also when profiling excess toxicity prior to clinical studies.

\section{Selective responses for clinical translation}

In clinical applications, it is not enough to show sufficient synergy or efficacy alone but also to evaluate the degree of potential side-effects of multi-targeting therapies to avoid selecting broadly toxic mono- or combinatorial therapies (Pemovska et al. 2013). To that end, we recently developed a computational-experimental approach, so-called drug combination prediction and testing (DCPT) platform, which makes use of proteome-wide compound-target interaction networks to narrow down the combinatorial explosion of potential combinations (more than 20,000 pairwise combinations were evaluated in this study, He et al. 2018). Importantly, the DCPT platform makes not only use of patient- 
derived samples to identify patient-specific synergistic combinations but also blood mononuclear cells from healthy individuals to avoid non-tolerable or overlapping toxicity profiles. As the first translational application, we predicted such selective combination responses for patients with $\mathrm{T}$ cell prolymphocytic leukemia ( $\mathrm{T}$ PLL), a rare but aggressive cancer, for which conducting large-scale clinical trials is difficult. DCPT platform successfully identified selective synergistic combinations individually for the T-PLL patients, each presenting with different resistance patterns and synergy mechanisms (He et al. 2018).

Off-target binding often causes collateral alterations in unintended biological pathways leading to toxicity. Such off-target pathway effects may vary greatly in different cell and tissue types, due to the context-specific expression of target-network proteins, leading to variable drug-induced toxicity. Attempts are being made to systematically model such drug off-target expression programs as a means to deprioritize potentially toxic compounds in the pre-clinical screening stage. For example, Gayvert et al. (2016) developed a computational model that utilizes target network connectivity and mRNA expression levels of primary and off-targets across 30 tissue types, along with structural features of molecules, with the aim to predict drugs failures in clinical trials due to unfavorable toxicity properties. However, the use of molecular profiles of off-targets to select patient-specific optimal therapy regimen remains still a distant goal. A major challenge is to collect systematic information about cell-specific off-target expression and its effect on drug toxicity at different concentrations. This knowledge gap is partly due to the lack of in vitro experimental systems that would enable toxicity estimation coupled with molecular profiling in order to identify safe therapeutic targets without the requirement of the lengthy animal in vivo experimentation.

\section{Model systems for off-target toxicity profiling}

Recently, cellular model systems, such as those based on human cardiomyocytes and hepatocytes derived from embryonic stem cells or induced pluripotent stem cells (iPSCs), have been investigated for cardiotoxicity and hepatotoxicity profiling as an alternative to animal models (Grimm et al. 2015; Sharma et al. 2017). However, the development of disease-relevant and cellspecific testing protocols is often time, as well as resource, consuming. Furthermore, the current experimental setups for toxicity assessment and offtarget identification using cell line models is not yet standardized enough to quickly assess thousands of compounds at different concentration ranges, partly explaining their limited use in high-throughput screening (HTS) setting. Patient-selective testing and clinical translation will also require compound and baseline molecular profiling of off-targets in both diseased and relevant controls cells, which is compromised by the currently limited access to control cells corresponding to the particular disease tissue types (e.g., liver, breast, pancreas, or kidney).

A particularly interesting and currently emerging model system for cancer research is based on patientderived organoid (PDO) models, in which normal and cancerous epithelial cells are grown together with immune cells, fibroblasts, adipocytes, and endothelial cells (Clevers and Tuveson 2019). Such living biobanks make it possible to carry out co-cultures of cancer organoids with non-neoplastic stromal cells to probe the tumor microenvironment. Accordingly, PDOs enable compound and molecular profiling in primary patient tumors and their surroundings, hence providing exciting possibilities for dissecting the cancer- and patient-specific mechanisms for precision therapies, including immunotherapies. For toxicity estimation, PDOs provide the opportunity for rapid off-target profiling and phenotypic validation studies in a model system that recapitulates in vivo mechanisms more closely than the standard cell culture models (Clevers and Tuveson 2019).

\section{Outlook for off-target modeling}

We argue that a careful dissection of parameters quantifying efficacy, toxicity, and synergy of multi-targeting mono- and combinatorial therapies in pre-clinical model systems, such as PDOs, is critical for developing safe and effective therapeutic regimens for cancers and other diseases. In addition to the in vitro or ex vivo model systems, there is also a need for flexible computational platforms that can speed up the early investigation of both the therapeutic and toxic effects of small molecules before entering into lengthy and costly animal or clinical studies (Ravikumar and Aittokallio 2018). Such in silico tools and databases should be computationally efficient to allow their implementation into an HTS pipeline, and easily usable also by non-computational researchers, to 
enable their wide application among the drug screening community. Proteome-wide mapping of both on- and off-target potencies of compounds is important for accurate drug response modeling, regardless whether it is done using machine learning algorithms or pre-clinical models. Due to the intensive efforts required for the compound-target bioactivity data annotation and harmonization, we welcome everyone to join our communitydriven drug target commons (DTC) initiative (https://drugtargetcommons.fimm.fi/). DTC provides a web-platform (database with a user interface) for opendata integration and standardization to enable comprehensive mapping, reuse, and analysis of compoundtarget interaction profiles for both speeding up and derisking of drug discovery and repurposing studies (Tang et al. 2018; Tanoli et al. 2019).

\section{References}

Clevers H, Tuveson DA. Organoid models for cancer research. Annu Rev Cancer Biol. 2019;3:223-34.

Gayvert KM, Madhukar NS, Elemento O. A data-driven approach to predicting successes and failures of clinical trials. Cell Chem Biol. 2016;23(10):1294-301.

Grimm FA, Iwata Y, Sirenko O, Bittner M, Rusyn I. High-content assay multiplexing for toxicity screening in induced pluripotent stem cell-derived cardiomyocytes and hepatocytes. Assay Drug Dev Technol. 2015;13(9):529-46.

He L, Tang J, Andersson EI, Timonen S, Koschmieder S, Wennerberg K, et al. Patient-customized drug combination prediction and testing for T-cell prolymphocytic leukemia patients. Cancer Res. 2018;78(9):2407-18.
Knight ZA, Lin H, Shokat KM. Targeting the cancer kinome through polypharmacology. Nat Rev Cancer. 2010;10(2): 130-7.

Kuenzi BM, Remsing Rix LL, Kinose F, Kroeger JL, Lancet JE, Padron E, et al. Off-target based drug repurposing opportunities for tivantinib in acute myeloid leukemia. Sci Rep. 2019;9(1):606.

Menden MP, Wang D, Mason MJ, Szalai B, Bulusu KC, Guan Y, et al. Community assessment to advance computational prediction of cancer drug combinations in a pharmacogenomic screen. Nat Commun. 2019;17;10(1):2674.

Pemovska T, Kontro M, Yadav B, Edgren H, Eldfors S, Szwajda A, et al. Individualized systems medicine strategy to tailor treatments for patients with chemorefractory acute myeloid leukemia. Cancer Discov. 2013;3(12):1416-29.

Pemovska T, Johnson E, Kontro M, Repasky GA, Chen J, Wells P, et al. Axitinib effectively inhibits BCR-ABL1(T315I) with a distinct binding conformation. Nature. 2015;519(7541):102-5.

Ravikumar B, Aittokallio T. Improving the efficacy-safety balance of polypharmacology in multi-target drug discovery. Expert Opin Drug Discov. 2018;13(2):179-92.

Sharma A, Burridge PW, McKeithan WL, Serrano R, Shukla P, Sayed $\mathrm{N}$ et al. High-throughput screening of tyrosine kinase inhibitor cardiotoxicity with human induced pluripotent stem cells. Sci Transl Med 2017 15;9(377).

Tang J, Tanoli Z-u-R, Ravikumar B, Alam Z, Rebane A, VähäKoskela M, et al. Drug target commons: a community effort to build a consensus knowledge base for drug-target interactions. Cell Chem Biol. 2018;25(2):224-9.

Tanoli Z, Alam Z, Ianevski A, Wennerberg K, Vähä-Koskela M, Aittokallio T. Interactive visual analysis of drug-target interaction networks using drug target profiler, with applications to precision medicine and drug repurposing. Brief Bioinform. 2019 (in press). https://doi.org/10.1093/bib/bby119.

Publisher's note Springer Nature remains neutral with regard to jurisdictional claims in published maps and institutional affiliations. 